\title{
Downregulation of Renal MRPs Transporters in Acute Lymphoblastic Leukemia Mediated by the IL-6/STAT3/PXR Signaling Pathway
}

Yue Zhou,' Ai-Qing Nie,' Shang Chen, ${ }^{2}$ Meng-Meng Wang,' Rui Yin, 'Bo-Hao Tang, 'Yue-E Wu,' Fan Yang,' Bin Du,' Hai-Yan Shi, ${ }^{3}$ Xin-Mei Yang, ${ }^{3}$ Guo-Xiang Hao,' Xiu-Li Guo, (D) ${ }^{4}$ Qiu-Ju Han, ${ }^{5}$ Yi Zheng, ${ }^{1} *$ Wei Zhao (D) ${ }^{1,3, *}$

'Department of Clinical Pharmacy, Key Laboratory of Chemical Biology (Ministry of Education), School of Pharmaceutical Sciences, Cheeloo College of Medicine, Shandong University, Jinan, People's Republic of China; ${ }^{2}$ Institute of Biochemical and Biotechnological Drug, Key Laboratory of Chemical Biology (Ministry of Education), School of Pharmaceutical Sciences, Cheeloo College of Medicine, Shandong University, Jinan, People's Republic of China; ${ }^{3}$ Department of Pharmacy, Shandong Provincial Qianfoshan Hospital, The First Affiliated Hospital of Shandong First Medical University, Jinan, People's Republic of China; ${ }^{4}$ Department of Pharmacology, Key Laboratory of Chemical Biology (Ministry of Education), School of Pharmaceutical Sciences, Cheeloo College of Medicine, Shandong University, Jinan, People's Republic of China; Institute of Immunopharmaceutical Sciences, Key Laboratory of Chemical Biology (Ministry of Education), School of Pharmaceutical Sciences, Cheeloo College of Medicine, Shandong University, Jinan, People's Republic of China

*These authors contributed equally to this work

Correspondence: Yi Zheng; Wei Zhao Department of Clinical Pharmacy, School of Pharmaceutical Sciences, Cheeloo College of Medicine, Shandong University, No. 44,

Wenhua West Road, Jinan, Shandong

Province, People's Republic of China

Tel/Fax+8653I 88383308

Email zhengyi@sdu.edu.cn;

zhao4wei2@hotmail.com
Purpose: Considering prior investigations on reductions of renal multidrug resistanceassociated protein (MRP) 2 and 4 transporters in mice with acute lymphoblastic leukemia (ALL), we sought to characterize the underlying mechanisms responsible for IL-6/STAT3/ PXR-mediated changes in the expression of MRP2 and MRP4 in ALL.

Subjects and Methods: ALL xenograft models were established and intravenously injected with methotrexate (MTX) of MRPs substrate in NOD/SCID mice. Protein expression of MRPs and associated mechanisms were detected by Western blotting and immunocytochemistry. Plasma concentrations of MTX were determined using high-performance liquid chromatography-tandem mass spectrometry (HPLC-MS/MS).

Results: Plasma IL-6 levels in patients with newly diagnosed ALL were increased compared to children with pneumonia. Similarly, plasma IL-6 levels in ALL, ALL-tocilizumab (TCZ, an IL-6 receptor inhibitor) and ALL-S3I-201 (a selective inhibitor of STAT3) mice were increased compared to the control group. The MRP2, MRP4, and PXR expression in HK-2 cells treated with IL-6 were decreased, whereas the p-STAT3 expression was significantly increased compared to the control group results. These results are consistent with clearance of MRPs-mediated MTX in the ALL group. These effects were attenuated by blocking IL-6/ STAT3/PXR signaling pathway.

Conclusion: Inflammation-mediated changes in pharmacokinetics are thought to be executed through pathways IL-6-activated pathways, which can facilitate a better understanding of the potential for the use of IL- 6 to predict the severity of adverse outcomes and the major implications on potential ALL treatments.

Keywords: disease-induced pharmacokinetic change, renal transporters, inflammatory cytokines, clearance

\section{Introduction}

Renal proximal tubules play a crucial role in salt absorbance, water dynamics, and in the mechanistic of organic anions such as glucose from the glomerular filtrate. Further, these tubules help to eliminate endogenous and exogenous waste products from the body. Of all known transporters that function in the kidney, multidrug resistance-associated protein (MRP) transporters MRP2/4 are essential. For example, MRP2/4 are major determinants of the apical efflux of organic anions into the tubular lumen, including $\beta$-lactams, antivirals, diuretics, NSAIDs, and methotrexate (MTX). ${ }^{1-4}$ Thus, MRP2/4 contributes to intracellular drug concentrations and chemotherapeutic effects. In prior research composed by some of us, kidney tissues 
from mice afflicted by acute lymphoblastic leukemia (ALL) exhibited decreased mRNA and protein levels of MRP2/4, as well as decreased efficiencies in the transporter functions. ${ }^{5}$ However, regulatory mechanisms of the MRP2/4 proteins remain unclear concerning ALLaffected mice kidneys.

The expression of drug transporters is subject to transcriptional regulation by nuclear receptors, which regulate the expression of their target genes by binding to DNA response elements present in target gene promoters. These DNA response elements can be recognized and activated by steroids, thyroid hormones, and many other lipophilic compounds. Pregnane $\mathrm{X}$ receptor (PXR, NR1I2) is a type of nuclear receptor considered a master xenobiotic receptor that coordinates the regulatory dynamics of expression of genes encoding drug-metabolizing enzymes and drug transporters as well as the detoxification and elimination of xenobiotics and endotoxins from the body. ${ }^{6}$ Activation of PXR by hormones, bile acids, or xenobiotics can lead to the expression induction of drug transporter genes, including MDR1, MRP2, MRP3, and OATP1B. ${ }^{7-9}$ Moreover, many genes suppressed by inflammatory stimuli (eg, Interleukin-6 (IL-6)) can be induced by activation of PXR. ${ }^{10}$ Hartmann et al found that significantly lower mRNA levels of Mrp2 were seen in vitro in IL-6 and IL-1 $\beta$-treated Hepa 1-6 cells compared to untreated controls. $^{11}$ Abualsunun et al demonstrated that IL-6 induced decreased Cypa3a, Abcbla, and Abcc3 expression in vivo. ${ }^{12}$ These results indicated significant potential involvement of PXR and inflammatory stimuli in the regulation of MRP2 and MRP4 in kidneys during ALL.

In several diseases, inflammation induces proinflammatory cytokine release, especially interleukins (eg, IL-6) as an acute phase response. These proinflammatory cytokines can affect the expression and activity of drug-metabolizing enzymes and membrane transporters. IL-6 is one of the key cytokines involved in the inflammatory response and a major regulator. Elevated levels of IL-6 were found in the systemic circulation of patients who have acute leukemia and has been postulated as a possible diagnostic and prognostic marker use in newly afflicted patients with acute leukemia. ${ }^{13-16}$ A substantial alteration of numerous metabolizing enzymes and transporters was observed in IL-6 treated rodents through the activation of PXR, demonstrating the importance of cytokines in the regulation of drug-body pharmacokinetics, such as for CYP3A4, BSEP, and MRP2. ${ }^{10,11,17}$ However, little is known about the involvement of PXR in the regulation dynamics of renal transporters during ALL. The Janus kinase (JAK)-signal transducer and activator of transcription (STAT) pathway have central roles in the antitumor immunity dynamics in tumor microenvironments consequent to elevated IL-6 levels. ${ }^{18,19}$ IL-6 binds to IL- 6 receptor- $\alpha$ (IL-6R $\alpha$ ) on the cell surface, triggering the formation of a hexametric signaling complex comprising a gp130 homodimer. These events result in activation of JAKs and, in turn, mediate phosphorylation of gp130, ultimately leading to the recruitment and activation of cytosolic STAT3, which then translocates into the nucleus. ${ }^{19}$ Indeed, STAT3 plays an essential role in cytokine-mediated induction of acutephase response genes in vivo. However, the potential involvement of the JAK/STAT3 pathway and PXR remains to be elucidated.

In the present study, we hypothesized that the elevated level of IL- 6 mediated the downregulation of renal expression and function of MRP2/4 in ALL. We predicted that increased IL-6 in plasma would result in increased activation of STAT3 and decreased activation of nuclear receptor PXR in vivo. Hence, our main objectives were to investigate the role of the nuclear receptor $\mathrm{PXR}$ in mediating changes in the expression of MRP2/4 transporters during ALL and to examine the underlying mechanisms responsible for IL-6-mediated transporters in ALL disease state.

\section{Materials and Methods}

\section{Patient Recruitment}

Between June 2018 and December 2019, patients <18 years with newly diagnosed ALL were recruited into our trial. The patients were recruited at the Children's Hospital of Hebei Province and the First Affiliated Hospital of Shandong First Medical University (Shandong Provincial Qianfoshan Hospital). ALL were diagnosed if $\geq 25 \%$ of the lymphoblasts were present in bone marrow (BM). Immunophenotypic and karyotypic features of leukemic cells were determined following standardized techniques. ${ }^{20,21}$ Cytogenetic 
assessments of TEL-AML1, BCR-ABL1, E2A-PBX1, and MLL rearrangement were facilitated through karyotyping, fluorescence in situ hybridization (FISH) and/or polymerase chain reaction (PCR) methods. ${ }^{22}$ A complete blood count was performed upon new diagnoses of conditions by using a cell-counting device (Pentra 120 counter, HORIBA, France). Children with suspected or confirmed community-acquired pneumonia were included as controls. After routine biochemical and microbiological tests were performed, the remaining blood specimens were collected. Peripheral blood samples were centrifuged (4000 rpm for $10 \mathrm{~min}$ ) post-collection, and the obtained plasma was kept at $-80{ }^{\circ} \mathrm{C}$ prior to any analyses. Cytokine profiles in plasma were performed using the Bio-Plex human cytokines 27-plex (R\&D Systems). Range quality control standards provided by the manufacturer were assayed on each plate. All values were analyzed in duplicate and are reported in units of $\mathrm{pg} / \mathrm{mL}$. All aspects of the design and experimentation were reviewed and approved by the Institutional Ethics Board of the Children's Hospital of Hebei Province and the First Affiliated Hospital of Shandong First Medical University (Shandong Provincial Qianfoshan Hospital). This study was conducted in accordance with the Declaration of Helsinki. We obtained informed consent from parents or guardians of all enrolled patients.

\section{Cell Culture}

Human acute T-cell lymphoblastic leukemia cells (CCRF-CEM) and human tubular epithelial cells (HK2) were purchased from the Cell Bank of the Chinese Academy of Sciences (Shanghai, China). CCRF-CEM cells were maintained in RPMI 1640 medium (GIBCO, USA) supplemented in $10 \%$ heat-inactivated fetal bovine serum (GIBCO, USA), antibiotics (containing $10,000 \mathrm{U} / \mathrm{mL}$ of penicillin and $10 \mathrm{mg} / \mathrm{L}$ of streptomycin), $2 \mathrm{mM}$ of L-glutamine, $1 \mathrm{mM}$ of sodium pyruvate, $4.5 \mathrm{~g} / \mathrm{L}$ of glucose, and $10 \mathrm{mM}$ of HEPES, at $37{ }^{\circ} \mathrm{C}$ and in a humidified atmosphere $\left(5 \% \mathrm{CO}_{2}, 95 \% \mathrm{O}_{2}\right)$. HK-2 cells were cultured in K-SFM (Invitrogen, USA) with both cell growth supplement and 10\% heatinactivated fetal bovine serum added, followed by incubation at $37{ }^{\circ} \mathrm{C}$ in a humidified atmosphere $(5 \%$
$\mathrm{CO}_{2}, 95 \% \mathrm{O}_{2}$ ). All experiments were conducted with cells within 3-6 passages.

\section{ALL Xenograft Models}

The 6-week-old immunocompromised male NOD. CB17/scid Arc (NOD/SCID) mice were purchased from Peking University Resources Centre (Beijing, China). Mice were maintained at a constant temperature-controlled room $\left(25 \pm 2{ }^{\circ} \mathrm{C}\right)$ with a 12-hour lightdark cycle. Mice were allowed water and food ad libitum and were acclimated for a minimum period of 1 week before experimentation. Animals were sublethally irradiated ( $1 \mathrm{~Gy} / \mathrm{min}$, total $2 \mathrm{~Gy}$ ) and randomly distributed into six treatment groups $(\mathrm{N}=6$ each group), including the control group, ALL, and ALLS3I-201 group treated with an intraperitoneal dose of $10 \mathrm{mg} / \mathrm{Kg} /$ day S3I-201 (the selective STAT3 inhibitor, Sigma-Aldrich, USA) ${ }^{23}$; control group, ALL group, and ALL-tocilizumab (TCZ) group treated with an intraperitoneal dose of $10 \mathrm{mg} / \mathrm{Kg}$ TCZ (the IL-6 receptor inhibitor, Roche Ltd, Switzerland) weekly for 4 weeks. $^{24,25}$ The ALL and normal mice were intravenously injected with $1 \times 10^{6}$ cells and an equivalent volume of saline via the tail vein, respectively. Mice were monitored and weighed regularly post-inoculation with the tumor-afflicted cells. Disease status was determined by detections of human $\mathrm{CD}^{+} 5^{+}$cells in peripheral blood using flow cytometry (Epics-XLII, Beckman, USA). Proportions of human $\mathrm{CD} 45^{+}$cells in peripheral blood were $>5 \%$, which we considered a successful marker for ALL xenograft models. ${ }^{5}$ Animal care and experimental procedures complied with the Institutional Guidelines of the Animal Care and Use Committee at Shandong University (Shandong, China). This project was conducted at the School of Pharmaceutical Sciences, Shandong University, with approvals granted by the Animal Ethics Committee of Shandong University.

\section{Western Blotting Analyses}

HK-2 cells $\left(5 \times 10^{5}\right.$ per well $)$ were seeded in 6-well plates and cultured until $80-90 \%$ confluence, whereafter they were exposed to $10 \mathrm{ng} / \mathrm{mL}$ IL-6 (R\&D Systems, USA) for $24 \mathrm{~h}$. Cells were then harvested and analyzed by Western blotting. In addition, HK-2 
cells $\left(5 \times 10^{5}\right.$ per well $)$ were transfected with $100 \mathrm{nM}$ of siRNAs for STAT3 or PXR (GenePharma, China) for $8 \mathrm{~h}$ following the manufacturer's protocols. The siRNA oligonucleotides were synthesized by Sangon Biotech (Shanghai, China), with the following sequences: 5'-GGACGACUUUGAUUU CAACTT-3' and 5'-GUUGAAAUCAAAGUCG UCCTT-3' for STAT3; 5'-CCUUGAUCAAGCGGAAGAATT-3' and 5'-UUCUUCCGCUUGAUCAAGGTT-3' for PXR. Cell cultures were then switched to a new medium for an additional $24 \mathrm{~h}$ before treating cells with IL-6 (10 ng/ $\mathrm{mL}$ ) for another $24 \mathrm{~h}$. These cells were also analyzed by Western blotting. Membrane proteins were extracted from HK-2 and kidney cells using Minute Plasma Membrane Isolation Kits following the manufacturer's protocols (Invent Biotechnologies, USA). Nuclear proteins were extracted using nuclear protein isolation kits (Solarbio, China) following the manufacturer's protocols. Measures for total protein were determined using Pierce Rapid BCA Protein Assay Kits (Thermo Fisher Scientific, USA) following the manufacturer's protocols. Samples of protein were heated up to $100{ }^{\circ} \mathrm{C}$ for $5 \mathrm{~min}$ prior to loading in acrylamide gels for sodium dodecyl sulfate-polyacrylamide gel electrophoresis (SDS-PAGE). Proteins were transferred to a polyvinylidene fluoride membrane (Millipore, USA), and blots were blocked overnight with 5\% skimmed milk in tris-buffered saline tween-20 (TBST). Target proteins were detected using primary antibodies antiMRP2 (PA5-86719, Invitrogen, USA), anti-MRP4 (MA1-35682, Invitrogen, USA), anti-STAT3 (ab68153, Abcam, USA), anti-p-STAT3 (ab76315, Abcam, USA), and anti-PXR (PA5-102431, Invitrogen, USA). $\mathrm{Na}^{+} / \mathrm{K}^{+}$ ATPase (ab76020, Abcam, USA) and $\beta$-actin (ab179467, Abcam, USA) were used as probes in the same blots as respective proteins. Secondary antibodies used included either goat anti-rat (ab6734, Abcam, USA) or goat anti-rabbit IgG (ab205718, Abcam, USA) based upon the primary antibody used. Antibody binding was detected by enhanced chemiluminescence reagent (Millipore, USA) and quantified by densitometry using a ChemiDoc XRS + molecular imager (Bio-Rad, USA). Data were expressed as relative densities of proteins normalized to $\mathrm{Na}^{+} / \mathrm{K}^{+}$ATPase or $\beta$-actin. Triplicated samples were themselves run in triplicate in experiments.

\section{Pharmacokinetic Analysis}

Post-successful establishment of ALL xenograft models, mice were intravenously injected with a single dose of $50 \mathrm{mg} / \mathrm{Kg}$ of MTX (substrate of MRP2 and MRP4) via the tail vein. ${ }^{5}$ Animals were anesthetized with isoflurane. Blood samples were collected at $5 \mathrm{~min}$, $2 \mathrm{~h}$, and $4 \mathrm{~h}$ from the retroorbital sinus of each mouse. Thereafter, the blood samples were immediately centrifuged at $4000 \mathrm{rpm}$ for $10 \mathrm{~min}$ ) and the obtained plasma was kept at $-80{ }^{\circ} \mathrm{C}$ before subsequent pharmacokinetic analyses. Then, all mice were rapidly euthanized and carefully excised kidneys were kept at $-80^{\circ} \mathrm{C}$ or fixed in $4 \% p$-formaldehyde prior to further analyses. Plasma concentrations of MTX were determined using highperformance liquid chromatography-tandem mass spectrometry (HPLC-MS/MS) (AB SCIEX, USA). Inter and intra-day coefficients of variation (CVs) were all $<10 \%$. Detailed methods for substrate analyses were based upon those validated in previous studies. ${ }^{26}$ A non-compartmental model facilitated the characterization of the pharmacokinetics of methotrexate. Concentrations of plasma IL-6 were measured using ELISA kits following the manufacturer's protocols.

\section{Histology and Immunofluorescence Analysis}

Excised kidney tissues were rinsed with cold PBS, fixed overnight in $4 \% p$-formaldehyde for paraffin embedding, and $5 \mu \mathrm{m}$ sections were cut. For histology, kidney tissue slides were stained with H\&E. For immunofluorescence, slides were dried at $60^{\circ} \mathrm{C}$ for $1 \mathrm{~h}$, deparaffinized, and treated with three changes of xylene, each for $10 \mathrm{~min}$. Next, a graded series of alcohol from $100 \%$ to $50 \%$ was applied, followed by water and TBS. Heat-induced antigen retrieval was performed, and endogenous peroxidase activity was blocked using a peroxidaseblocking reagent. Slides were incubated with primary antibodies in a humidified chamber overnight at $4^{\circ}$ $\mathrm{C}$, followed by incubations with secondary antibody (goat antirabbit IgG, ab6717, Abcam, USA; goat anti-rat IgG, ab7093, Abcam, USA) diaminobenzidine, and hematoxylin for $30 \mathrm{~s}$, at room temperature. Finally, $\geq 10$ randomly chosen fields within each section were photographed and assessed using an optical microscope at a $400 \times$ magnification (Olympus Corporation, Japan). 
Table I Demographic Data of Patients with ALL and Pneumonia

\begin{tabular}{|l|c|c|}
\hline Characteristic & ALL Patients (n = 18) & Pneumonia Patients (n = I2) \\
\hline Sex & & 7 \\
Male, $n$ (\%) & 8 & 5 \\
Female, $n$ (\%) & 10 & $5.5(3.6-10.4)$ \\
Median age, years (range) & $5.7(2.9-11.5)$ & $26.7(16.1-43.3)$ \\
Median body weight, kg (range) & $25.9(11.4-44.1)$ & $6.1(3.6-14.9)$ \\
Median white blood cells, $\times 10^{9} /$ L (range) & $39.3(5.1-319.1)$ & \\
\hline Immunologic subtype & & \\
B cell, $n$ (\%) & $14(77.78)$ & \\
T cell, $n$ (\%) & $4(23.22)$ & \\
\hline
\end{tabular}

\section{mRNA Analyses}

Total RNA was extracted from HK-2 cells and tissue samples using a one-step method following the manufacturer's protocols for a TRIzol kit (Invitrogen, USA). Concentrations and qualities of RNA samples were determined based upon absorbance values at 260 and $280 \mathrm{~nm}$ measured by a Nanodrop One (Thermo Fisher Scientific, USA). Reverse transcription was performed following the manufacturer's protocols for a reverse transcription kit (Thermo Fisher Scientific, USA). Oligonucleotide primer sets used in this study were constructed by and purchased from Sangon Biotech. Subsequently, relative mRNA expression was measured using a fluorescent quantitative PCR kit (Takara, Japan) and a CFX96 Touch real-time PCR detection system (Bio-Rad, USA). Gene expression analyses were performed using the $2^{-\Delta \Delta \mathrm{CT}}$ threshold cycle $\left(\mathrm{C}_{\mathrm{T}}\right)$ method, employing the glyceraldehyde 3-phosphate dehydrogenase (GAPDH) gene as the endogenous gene. Ensuing results were expressed as relative mRNA levels.

\section{Data and Statistical Analysis}

Statistical analysis was carried only for groups with a group size of $n \geq 5$ and the experimental groups were designed accordingly, using randomization. The levels of IL-6 in human and mice were performed using one-way analysis of variance (ANOVA) with follow-up unpaired $t$-test. Relative protein semiquantification was performed using AlphaEaseFC software (AlphaInnotech, USA). The protein and mRNA expression were analyzed using unpaired $t$-test. Pharmacokinetic parameters were best described with noncompartmental analyses (WinNolin 6.3, USA). Statistical analyses were completed using the Statistical Package for Social Sciences (SPSS Version 13.0 for Windows, IBM, USA).

\section{Results}

\section{Patient's Characteristics}

Eighteen patients with newly diagnosed ALL were enrolled. Of these, 14 were B-cell ALL afflicted. The median patient age was 5.7 years (range 2.9 to 11.5 years). The median white blood cells $(\mathrm{WBCs})$ count was $=39.3 \times 10^{9} / \mathrm{L}$ (range 5.1 to $319.1 \times 10^{9} / \mathrm{L}$ ). As a control group, 12 children with suspected or confirmed community-acquired pneumonia were enrolled. For these patients, the median age was 5.5 years (range $3.6-10.4$ years). The median WBCs counts was $=6.1 \times 10^{9}$ / L (range 3.6-14.9 $\times 10^{9} / \mathrm{L}$ ). Patient characteristics are presented in Table 1.

\section{IL-6 Levels Increased in ALL-Afflicted Patients and Animals}

Cytokine profiles in plasma were performed using the BioPlex human cytokines 27-plex. As shown in Figure 1, L-1 $\beta$, IL-6, IL-8, IL-10, IL-1, eotaxin, G-CSF, GM-CSF, IFN- $\gamma$, CXCL-10, MCP-1, and TNF- $\alpha$ were significantly higher in ALL-afflicted patients than patients with pneumonia. Furthermore, IL-6 levels for ALL-afflicted patients $(15.76 \pm 13.49 \mathrm{pg} / \mathrm{mL})$ mostly increased by 7.44 -fold than for patients with pneumonia $(2.12 \pm 1.71 \mathrm{pg} / \mathrm{mL}, p<$ $0.001)$. In NOD/SCID mice, plasma levels of IL-6 in the 
A

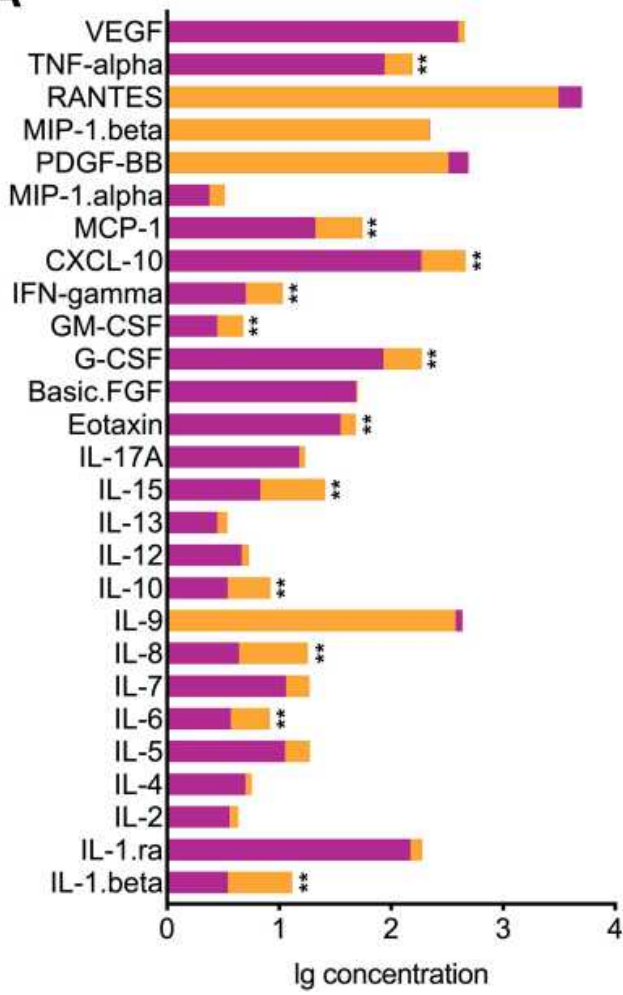

B

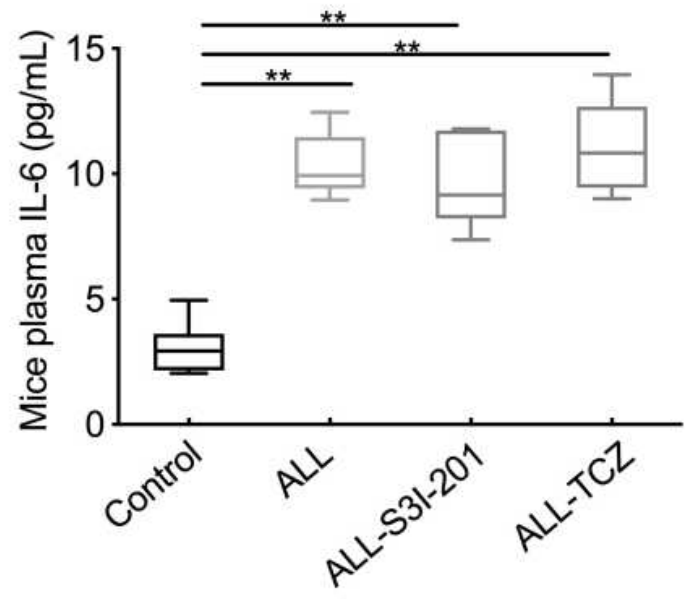

Figure I Plasma of IL-6 levels in ALL-afflicted patients and mice. (A) Plasma IL-6 levels in ALL and pneumonia-afflicted patients, as determined by Bio-Plex human cytokines 27-plex. ${ }^{* *} p<0.0 \mathrm{I}, \mathrm{ALL}$-afflicted patients versus pneumonia-afflicted patients. (B) Plasma IL-6 levels in the control, ALL, and ALL treated with S3I-20I and TCZ mice groups, as determined by ELISA. ${ }^{* *} p<0.01$ versus the control group.

ALL group, the ALL-TCZ and ALL-S3I-201 group (10.33 $\pm 1.26,9.6 \pm 1.81$ and $11.08 \pm 2.82 \mathrm{pg} / \mathrm{mL}$ ) increased respectively 3.41-fold, 3.17-fold and 3.64-fold compared with the control group $(3.04 \pm 1.05 \mathrm{pg} / \mathrm{mL})$. No obvious differences between ALL group, ALL-S3I-201 and ALLTCZ group were observed for plasma levels of IL-6 (Figure 1).

\section{IL-6 Suppressed the Expression of MRP2 and MRP4 Through the STAT3/PXR Pathway in HK-2 Cells}

To observe the effects of IL-6 upon the expression of MRP2 and MRP4 in HK-2 cells, HK-2 cells were exposed to $10 \mathrm{ng} / \mathrm{mL}$ of IL-6 for subsequent Western blotting analyses. Results indicated that p-STAT3 expression in HK-2 treated cells with IL-6 significantly increased by 1.56-fold compared with the control group. The presence of IL- 6 decreased the expression of PXR, MRP2, and MRP4 by $54.6 \%, 46.3 \%$, and $52.4 \%$ compared with respective levels for the control group (Figure 2A and B). Furthermore, to determine the role of the STAT3/PXR pathway in inducing adverse effects of IL-6 upon the expression of MRP2 and MRP4, HK-2 cells were treated with STAT3 and $P X R$ siRNA. As shown in Figure $2 \mathrm{~A}, \mathrm{C}$, and $\mathrm{D}$, when the STAT3/PXR pathway was inhibited by siRNA suppression of STAT3 or PXR, the effect of IL-6 upon suppression of MRP2 and MRP4 expression was notably attenuated (Supplementary Data Figure 1). Moreover, relative $M R P 2$ and MRP4 gene expression in HK-2 cells were detected by using quantitative polymerase chain reaction. As presented in Figure 2E, qRTPCR analysis of HK-2 cells indicated a significant decrease in $P X R, M R P 2$, and MRP4 in HK-2 cells 
A

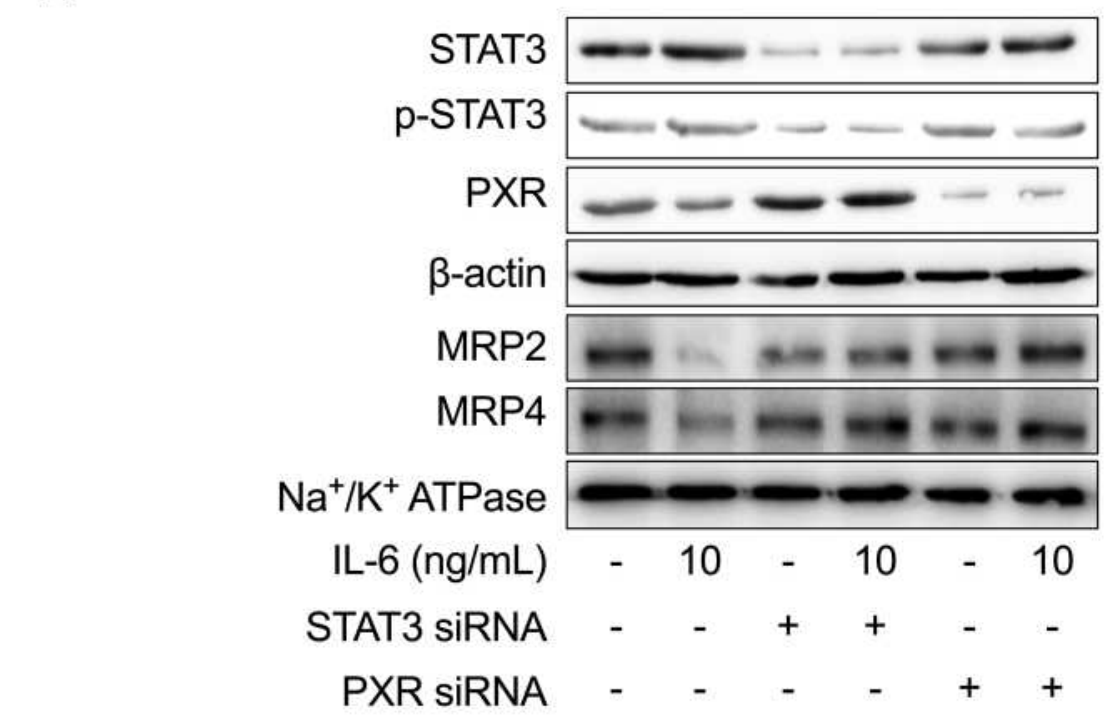

B
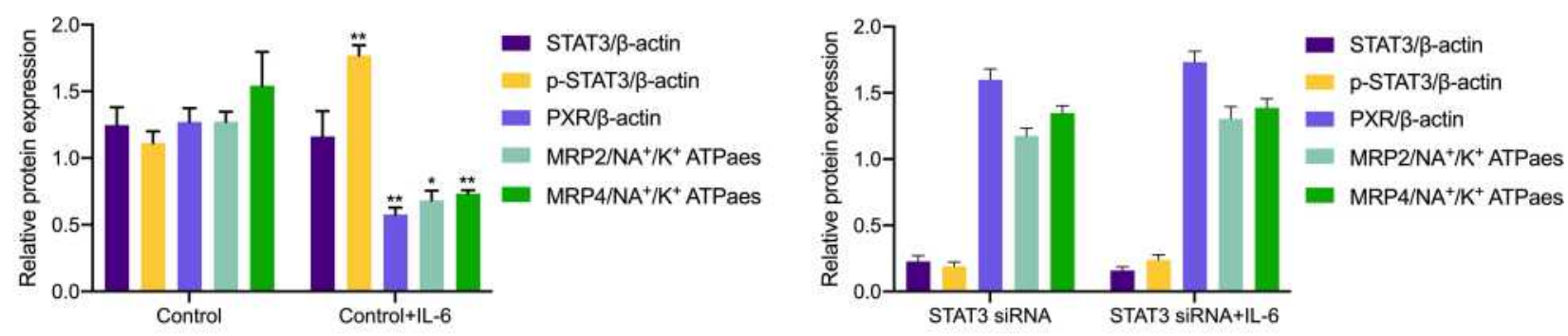

D

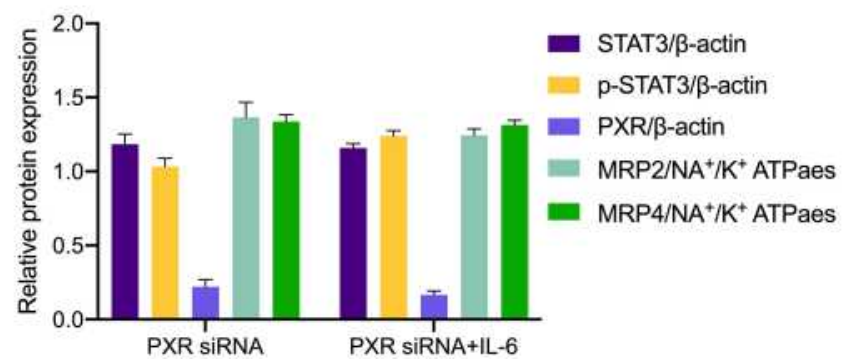

E

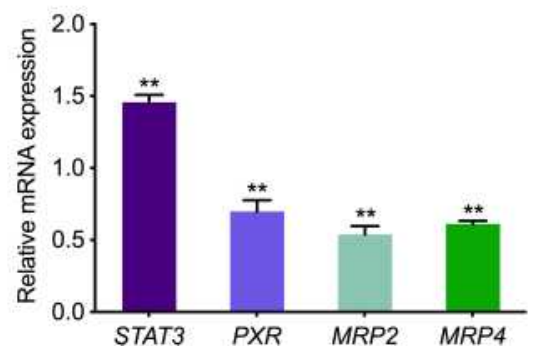

Figure 2 Expression of MRPs transporters in HK-2. (A) HK-2 cells were exposed to $10 \mathrm{ng} / \mathrm{mL}$ IL-6 with STAT3 and PXR siRNA for subsequent Western blotting. (B-D) Protein expression of targeted transporters was quantified and is viewed in the bar graphs. ${ }^{*} p<0.05$, $* * p<0.01$, versus control group. (E) The fold change with respect to mRNA expression of STAT3, PXR, MRP2, and MRP4 in HK-2 cells treated with IL-6 $(10 \mathrm{ng} / \mathrm{mL})$ compared to HK-2 cells. **p < 0.01 , versus control group.

treated with IL-6 compared with the control group. An increase in STAT3 was detected in HK-2 cells treated with IL-6. These results suggested that IL-6 was dependent upon the STAT3/PXR pathway to suppress the expression of MRP2 and MRP4 in HK-2-afflicted cells.

\section{IL-6 Regulates the Pharmacokinetics of MTX Through STAT3/PXR/MRP2/4 Pathway in NOD/SCID Mice}

The effect of IL-6 upon the pharmacokinetics of MTX (MRP2 and MRP4 substrate) was evaluated via the use of Tocilizumab of IL-6 receptor inhibitor and S3I-201 

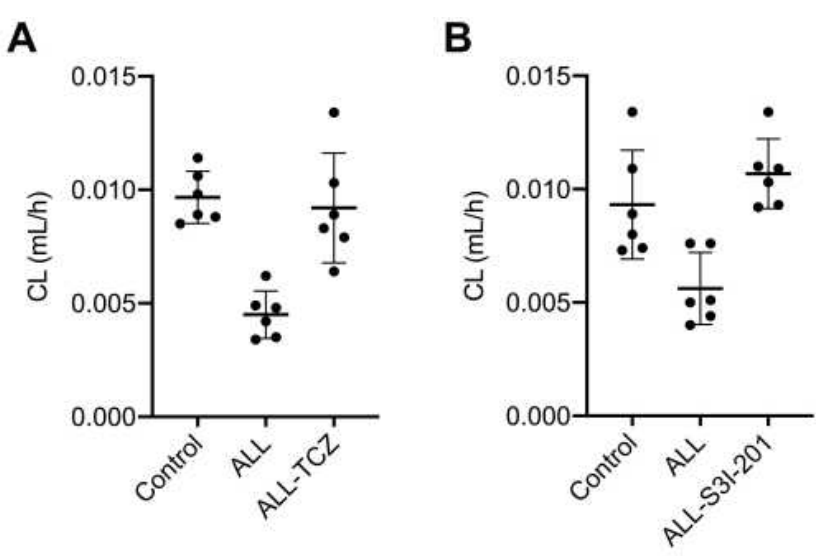

C

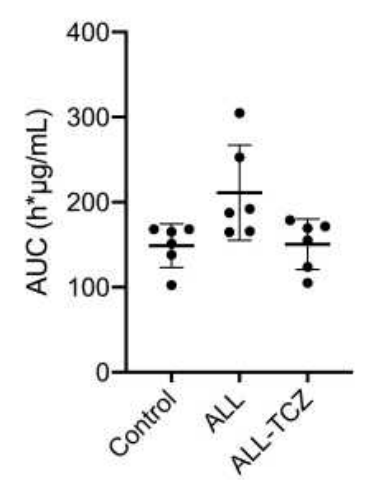

$\mu \mathrm{g} \mathrm{h} \mathrm{mL}^{-1}$ ) compared with the control group (148.88 \pm $25.65 \mu \mathrm{g} \mathrm{h} \mathrm{mL}{ }^{-1}, p=0.042 ; 139.74 \pm 38.09 \mu \mathrm{g}$ $\mathrm{h} \mathrm{mL}{ }^{-1}, p=0.024$;) and the ALL-TCZ group and ALLS3I-201 group $\left(150.63 \pm 29.65 \mu \mathrm{g} \mathrm{h} \mathrm{mL}^{-1}, p=0.049\right.$; $\left.123.96 \pm 13.18 \mu \mathrm{g} \mathrm{h} \mathrm{mL}^{-1}, p=0.046\right)$, respectively. No obvious differences between the control, the ALL-TCZ, and the ALL-S3I-201 groups were observed concerning the clearance and $\mathrm{AUC}_{0-\infty}$ of MTX (Figure $3 \mathrm{C}$ and D).

\section{IL-6 Suppressed the Expression of MRP2 and MRP4 Through the STAT3/PXR Pathway in NOD/SCID Mice}

To investigate the effect of IL-6 upon the expression of MRP2 and MRP4, the relative expression between all three treatment groups was detected using Western blotting. ALL group showed to decrease the MRP2 and MRP4 expressions compared with the control and the ALL-TCZ groups. In addition, the expression of PXR in ALL group was decreased compared with the control and the ALL-TCZ groups (Figure 4 and Supplementary Data Figure 2). Relative expression of Mrp2, Mrp4, and Pxr genes in NOD/SCID-afflicted mice was assessed. ALL group showed decrease Mrp2, Mrp4, and Pxr mRNA expression with numbers recorded as $23.08 \%, 43.40 \%$, and $29.45 \%$, and $22.90 \%$, $43.22 \%$, and $26.68 \%$ compared with the control and the ALL-TCZ groups (Figure 4C), respectively. As shown in Figure 5 and Supplementary Data Figure 3, protein expressions for both MRP2 and MRP4 were significantly decreased in kidney samples from the ALL group, compared with respective values for the control and the ALL-S3I-201 groups. qRT-PCR analyses of kidney tissues from NOD/SCID mice indicated significantly decreased levels in Mrp2, Mrp4, and Pxr with numbers recorded as $26.11 \%, 41.83 \%$, and $27.73 \%$, and $24.12 \%, 36.92 \%$, and $22.27 \%$ in the ALL group compared with the control and ALL-S3I-201 group, respectively (Figure 5C). Mrp2, Mrp4, and Pxr mRNA levels exhibited no differences for comparisons between the control, ALL-TCZ, and the ALL-S3I-201 groups. Expression of PXR was also decreased compared to the control and ALL-S3I-201 groups. PXR, MRP2, and MRP4 expression were similar between the control, ALL-TCZ, and the ALL-S3I-201 groups. These results were correspondent with the 
A

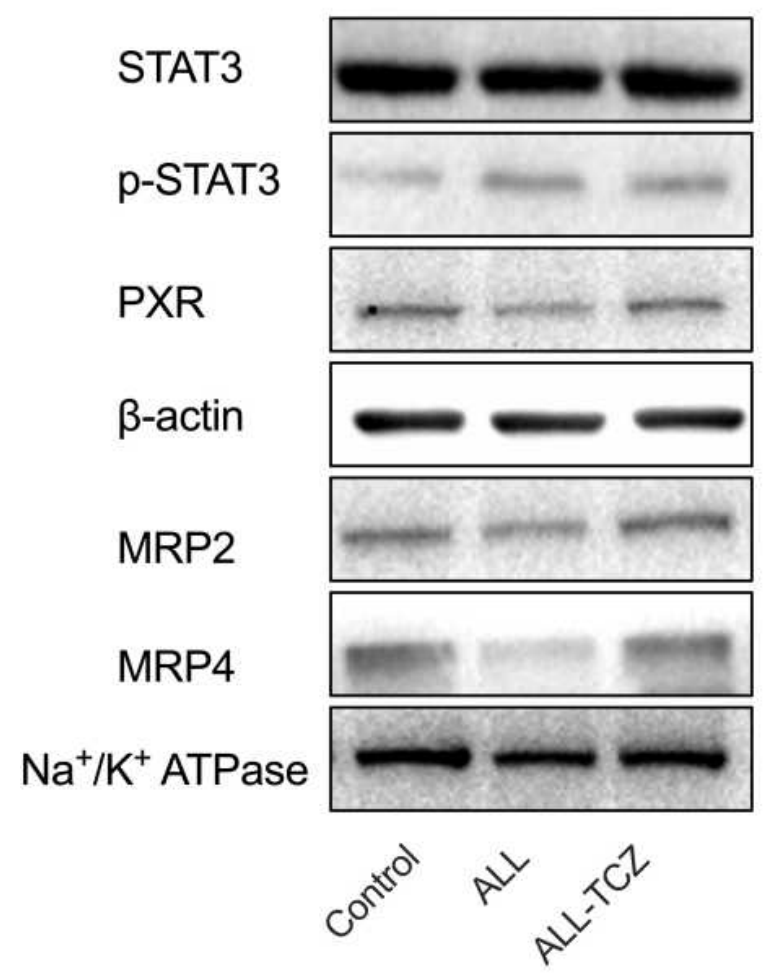

B

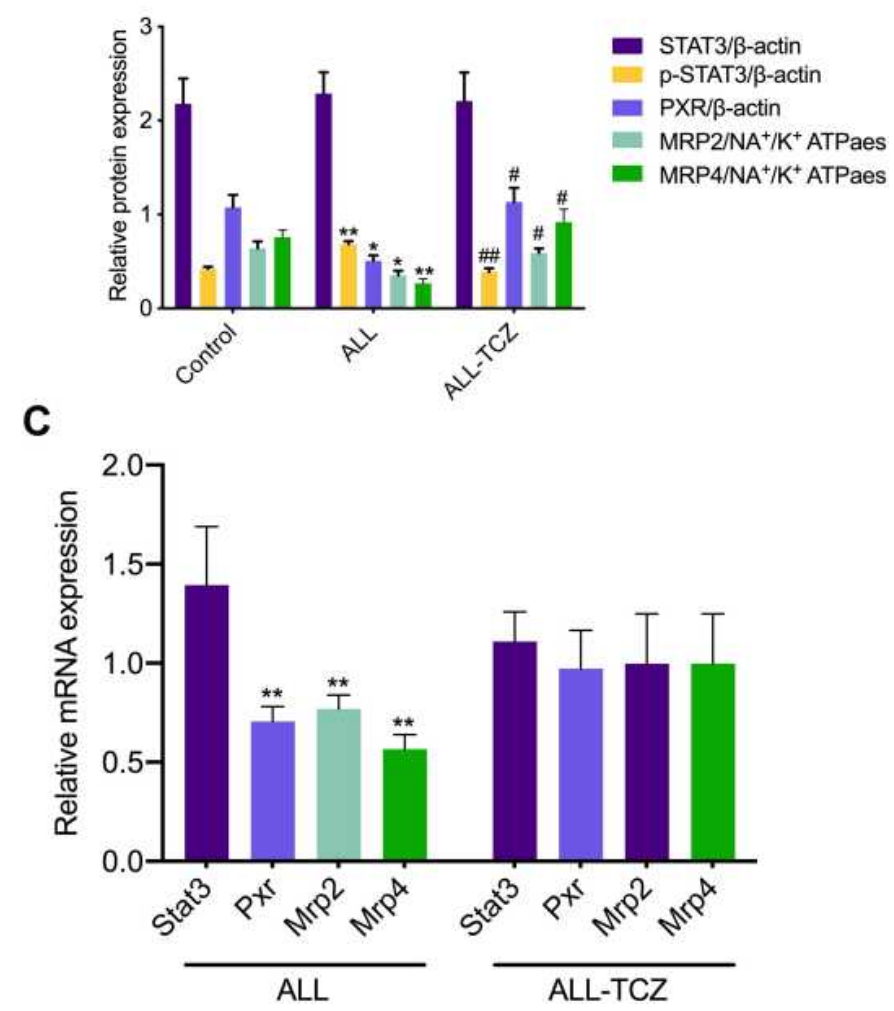

Figure 4 Expression of targeted renal proteins and mRNA in NOD/SCID mice treated with Tocilizumab. (A) Expression of target proteins in NOD/SCID mice detected using Western blotting. (B) Expression of targeted proteins was quantified and can be seen in the bar graphs. ${ }^{*} p<0.05,{ }^{* *} p<0.01$, versus the control group; ${ }^{\#} p<0.05$, ${ }^{\# \#} p<$ 0.01 , the ALL-TCZ group versus the ALL group. (C) The fold change with respect to mRNA expression of Stat3, Pxr, Mrp2, and Mrp4 in NOD/SCID mice. * $p<0.05$, **p < 0.01 , versus the control group.

immunohistochemistry analysis (Figure 6). In parallel with kidney function assessments, histological results based upon H\&E staining of kidneys indicated a lack of significant differences in kidney histological structure among comparisons of the six groups (Figure 7).

\section{Discussion}

Reduced functions for transports in the kidney tissues can play critical roles in determining intracellular drug concentrations and expected chemotherapeutic effects. Nuclear receptor PXR is crucial in regulating genes responsible for essential parts of the dynamics underlying the transporters. Moreover, there has been some knowledge gained from multi-analytical determinations of pro-inflammatory cytokines known to affect the expression and activity of membrane transporters. ${ }^{27}$ IL-6 is one such type of major proinflammatory cytokine and acts to modulate transporter levels by activating the STAT3 signaling pathway. Thus, this study's goal was to examine and characterize the roles of nuclear receptor PXR in mediating changes in renal expression of MRP2 and MRP4 and assess the potential involvement of the IL-6/STAT3 signaling pathway in ALL. Our findings demonstrated that elevated IL-6 induced downregulation in renal MRP2 and MRP4 mRNA expression and associated proteins through activation of STAT3 and PXR in NOD/SCID ALL mice.

During inflammation, secretion of IL-6 by monocytes and macrophages can regulate several genes through activation of the JAK/STAT3 signaling pathway, including for drug transporters. ${ }^{27,28}$ Following IL6 stimulation, dimerization of JAK3 causes phosphorylation and translocation of STAT3 into the 
A

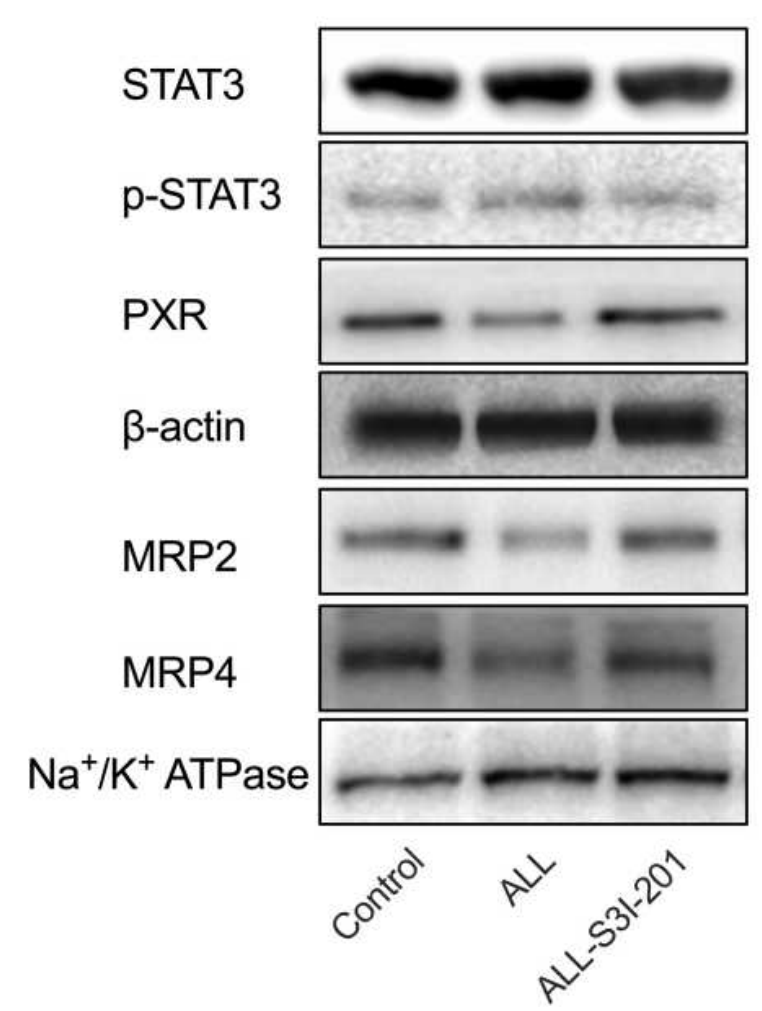

B
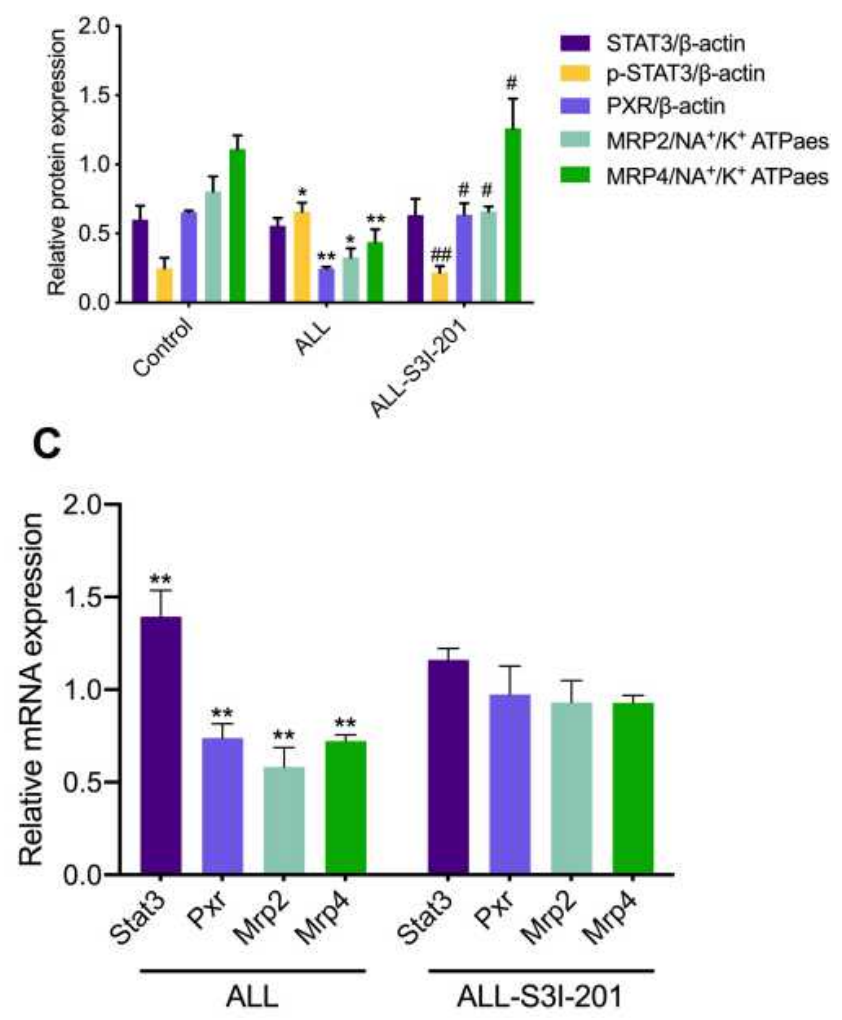

Figure 5 Expression of targeted renal proteins and mRNA in NOD/SCID mice treated with S3I-20I. (A) Expression of target proteins in NOD/SCID mice kidney detected using Western blotting. (B) Expression of targeted proteins was quantified and can be seen in the bar graphs. ${ }^{*} p<0.05,{ }^{* *} p<0.01$, versus the control group; ${ }^{*} p<0.05$, ${ }^{\#} p<$ 0.01 , the ALL-S3I-20I group versus the control group. (C) The fold change with respect to mRNA expression of Stat3, Pxr, Mrp2, and Mrp4 in NOD/SCID mice. *p < 0.05 , $*^{*} p<0.01$, versus the control group.

nucleus whereby it regulates transcription of numerous genes. In this study, elevated levels of IL-6 were found in the systemic circulation of mice afflicted by ALL and were linked to the downregulation of MRP2 and MRP4. IL-6 levels in newly diagnosed ALL patients were significantly increased (by 7.44-fold) than in patients with pneumonia. In NOD/SCID mice, we also found that IL-6 levels in the ALL group were significantly increased compared to the control group. These results indicated that IL- 6 might be an essential modulator in ALL. Some research has already clarified that IL-6 downregulated genes involved in drug elimination. ${ }^{29,30}$ Furthermore, we used HK-2 cells and NOD/SCID ALL-afflicted mice to explore mechanisms underlying IL-6 mediated regulation of MRPs transporters in the kidneys. Our results indicated that the expression of MRP2 and MRP4 transporters in the kidney were suppressed when HK-2 cells, as well as NOD/SCID ALL mice received IL-6 stimulation. We further explored the mechanisms influencing IL-6 downregulation of MRP2 and MRP4.

PXR is a key regulator of $\mathrm{ABC}$ drug transporters, including MDR1, MRP2, and BCRP. ${ }^{31}$ PXR is activated by endogenous cytokines (eg, Interleukin), ${ }^{32}$ and our results revealed that IL-6 induced downregulation of MRP2, MRP4, and PXR expression in HK2-afflicted cells and ALL mice. However, when PXR was siRNA-inhibited in HK-2 cells, the effect of IL-6 upon suppression of MRP2 and MRP4 expression was notably attenuated. These results revealed that IL-6 downregulated MRP2 and MRP4 expression via suppression of PXR. JAK/STAT3 signaling is a classic IL6-associated-pathway. To examine whether IL-6 directly affected MRP2 and MRP4 through STAT3, 


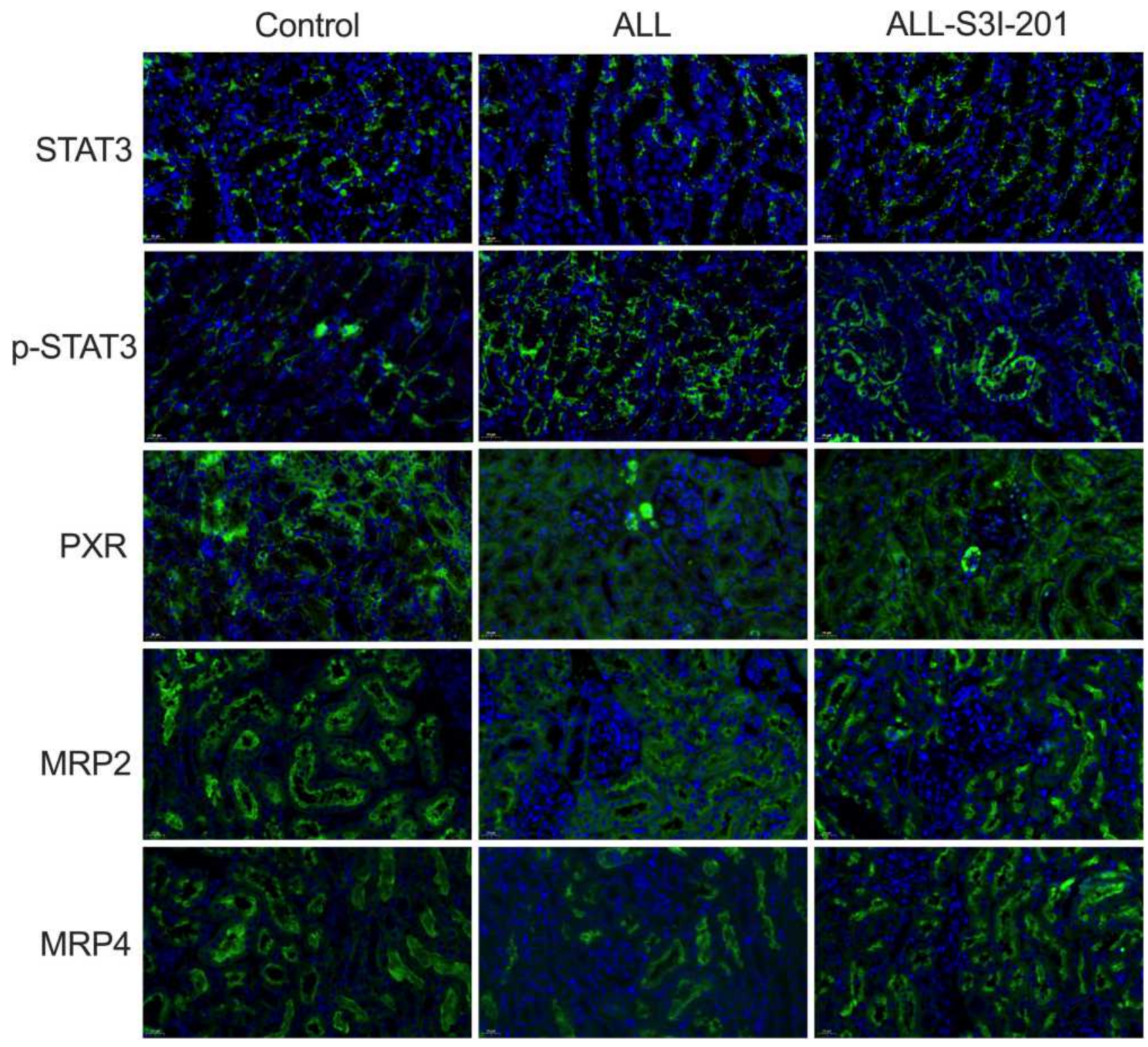

Figure 6 Immunohistochemical analysis for kidney tissues from NOD/SCID mice treated with S3I-20I. Immunohistochemical expression of target proteins in NOD/SCID mice kidney. Expression of STAT3, PXR, MRP2, and MRP4 proteins in the kidneys using immunocytochemistry. Original magnification $\times 400$. Arrowheads represent positive signals for transporter proteins.

we examined their relative expression levels in vivo and in vitro. As expected, STAT3 was activated from IL-6 treatments in HK-2 cells. When HK-2 cells were treated with STAT3 siRNA, the effect of IL-6 downregulation of MRP2, MRP4, and PXR was suppressed. Consistent with the in vitro results, we found that NOD/SCID mice treated with TCZ and S3I-201 had suppressed downregulation of MRP2, MRP4, and PXR through IL-6. Furthermore, we also examined the pharmacokinetics of MTX and substrates of MRP2 and MRP4 in NOD/SCID mice. MTX is primarily excreted into the urine in an unchanged form (at approximately $80-90 \%$ of the original dose), and renal handling involves tubular secretion and reabsorption. MTX is effluxed from cells across the luminal membrane and into the urine via MRP2 and MRP4. Therefore, MTX clearance was likely to have been mediated by MRPs. Pharmacokinetic data revealed that the elimination of MTX in ALL mice was suppressed compared with respective findings for control mice. When ALL mice were treated with TCZ and S3I-201, the effect of suppression was attenuated. Based on this observed 

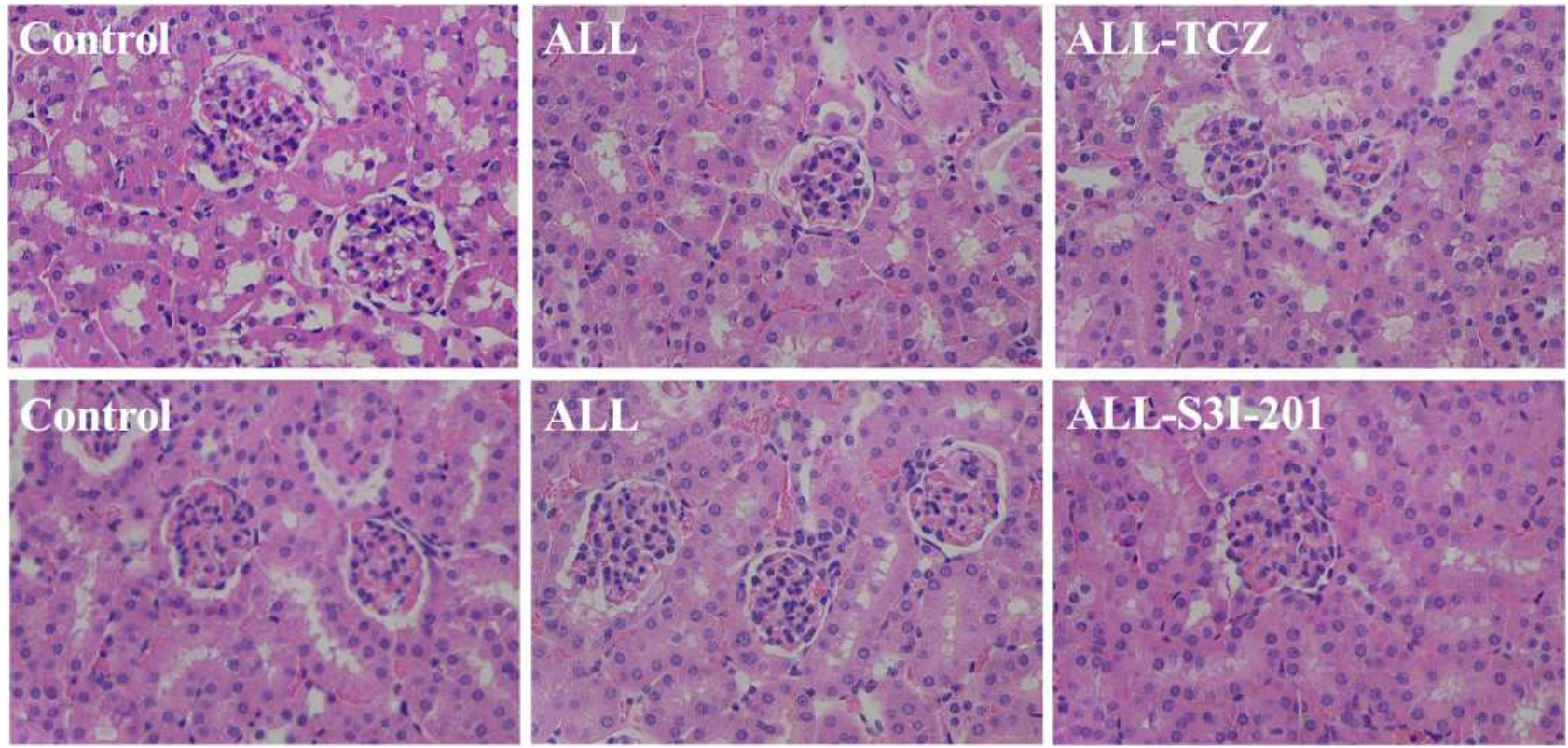

Figure 7 HE-staining analyses for kidneys from NOD/SCID mice treated with S3I-20I and Tocilizumab. H\&E staining of kidneys indicated a lack of significant differences in kidney histological structure among comparisons of the six groups NOD/SCID mice. Representative images of HE-stained kidney sections. Original magnification $\times 400$.

phenomenon, IL-6-mediated downregulated MRP2 and MRP4 transporters likely through a pathway downstream of STAT3 activation in the kidneys.

Due to the overlapping of substrates, a significant limitation in this study was the lack of specific substrates to evaluate the function of renal MRP2 and MRP4 transporters. MTX clearance is significantly affected by MRPs and OAT3 transporters. However, the effect of OAT3 transporter upon MTX clearance was not elucidated in this study and requires further investigation.

In conclusion, this study established and characterized the primary roles of the STAT3 signaling pathway had in influencing the dynamics of IL-6-mediated downregulation of MRP2 and MRP4 transporters in the kidneys, with partial involvement of PXR. As inflammation-mediated changes in pharmacokinetics are thought to be executed through IL-6-activated pathways, this information can facilitate the understanding of the potential for IL-6 to predict the severity of adverse outcomes and the major implications on potential ALL treatments.

\section{Author Contributions}

All authors made substantial contributions to conception and design, acquisition of data, or analysis and interpretation of data; took part in drafting the article or revising it critically for important intellectual content; agreed to submit to the current journal; gave final approval of the version to be published; and agree to be accountable for all aspects of the work.

\section{Funding}

This work was supported by National Science and Technology Major Projects for "Major New Drugs Innovation and Development" (2017ZX09304029-002), National Science Foundation of China (81703603), the Key Technologies R \& D Program of Shandong Province (2018GSF118053), Young Taishan Scholars Program of Shandong Province, Qilu Young Scholars Program of Shandong University.

\section{Disclosure}

Dr Wei Zhao reports grants, non-financial support from National Science and Technology Major Projects (2017ZX09304029-002), grants, non-financial support from National Science Foundation of China (81703603), grants, non-financial support from Young Taishan Scholars Program of Shandong Province, grants, non-financial support from Qilu Young Scholars Program of Shandong University. Dr Yi 
Zheng reports grants, non-financial support from the Key Technologies R \& D Program of Shandong Province (2018GSF118053), outside the submitted work. The authors declare no competing interests.

\section{References}

1. El-Sheikh AAK, Greupink R, Wortelboer HM, et al. Interaction of immunosuppressive drugs with human organic anion transporter (OAT) 1 and OAT3, and multidrug resistance-associated protein (MRP) 2 and MRP4. Transl Res. 2013;162(6):398-409. doi:10.1016/j. trs1.2013.08.003

2. Smeets PH, van Aubel RA, Wouterse AC, van den Heuvel JJ, Russel FG. Contribution of multidrug resistance protein 2 (MRP2/ABCC2) to the renal excretion of p-aminohippurate $(\mathrm{PAH})$ and identification of MRP4 (ABCC4) as a novel PAH transporter. J Am Soc Nephrol. 2004;15 (11):2828-2835. doi:10.1097/01.ASN.0000143473.64430.AC

3. Bakos E, Evers R, Sinko E, Varadi A, Borst P, Sarkadi B. Interactions of the human multidrug resistance proteins MRP1 and MRP2 with organic anions. Mol Pharmacol. 2000;57(4):760-768. doi:10.1124/mol.57.4.760

4. El-Sheikh AA, van den Heuvel JJ, Koenderink JB, Russel FG. Interaction of nonsteroidal anti-inflammatory drugs with multidrug resistance protein (MRP) 2/ABCC2- and MRP4/ABCC4-mediated methotrexate transport. J Pharmacol Exp Ther. 2007;320(1):229-235. doi:10.1124/jpet.106.110379

5. Zhou Y, Du B, Kan M, et al. Drug elimination alteration in acute lymphoblastic leukemia mediated by renal transporters and glomerular filtration. Pharm Res. 2020;37(8):158. doi:10.1007/s11095-020-02896-8

6. Oladimeji PO, Chen T. PXR: more than just a master xenobiotic receptor. Mol Pharmacol. 2018;93(2):119-127. doi:10.1124/mol.117.110155

7. Chai X, Zeng S, Xie W. Nuclear receptors PXR and CAR: implications for drug metabolism regulation, pharmacogenomics and beyond. Expert Opin Drug Metab Toxicol. 2013;9(3):253-266. doi:10.1517/17425255.2013.754010

8. Evers R, Piquette-Miller M, Polli JW, et al. Disease-associated changes in drug transporters may impact the pharmacokinetics and/or toxicity of drugs: a white paper from the international transporter consortium. Clin Pharmacol Ther. 2018;104(5):900-915. doi:10.1002/cpt.1115

9. Meyer Zu Schwabedissen HE, Kim RB. Hepatic OATP1B transporters and nuclear receptors PXR and CAR: interplay, regulation of drug disposition genes, and single nucleotide polymorphisms. Mol Pharm. 2009;6(6):1644-1661. doi:10.1021/mp9000298

10. Teng S, Piquette-Miller M. The involvement of the pregnane X receptor in hepatic gene regulation during inflammation in mice. $J$ Pharmacol Exp Ther. 2005;312(2):841-848. doi:10.1124/jpet.104.076141

11. Hartmann G, Cheung AK, Piquette-Miller M. Inflammatory cytokines, but not bile acids, regulate expression of murine hepatic anion transporters in endotoxemia. $J$ Pharmacol Exp Ther. 2002;303(1):273-281. doi:10.1124/jpet.102.039404

12. Abualsunun WA, Piquette-Miller M. STAT3 is involved in IL-6-mediated downregulation of hepatic transporters in mice. J Pharm Pharm Sci. 2018;21(1s):325s-334s. doi:10.18433/ jpps30241

13. El-Gohary GM, Azzam HM, Ahmed OI, El-Shokry MH. Proinflammatory cytokines and depression in patients with acute leukemia. Egypt J Immunol. 2008;15(1):13-24.

14. Yoon JY, Lafarge S, Dawe D, et al. Association of interleukin-6 and interleukin-8 with poor prognosis in elderly patients with chronic lymphocytic leukemia. Leuk Lymphoma. 2012;53(9):1735-1742. doi:10.3109/10428194.2012.666662
15. Soegaard SH, Rostgaard K, Skogstrand K, Wiemels JL, Schmiegelow K, Hjalgrim H. Neonatal inflammatory markers are associated with childhood B-cell precursor acute lymphoblastic leukemia. Cancer Res. 2018;78(18):5458-5463. doi:10.1158/00085472.CAN-18-0831

16. Horacek JM, Kupsa T, Vasatova M, Jebavy L, Zak P. Serum cytokine and adhesion molecule profile differs in newly diagnosed acute myeloid and lymphoblastic leukemia. Biomed Pap Med Fac Univ Palacky Olomouc Czech Repub. 2015;159(2):299-301. doi:10.5507/ bp. 2014.051

17. Yang Q, Doshi U, Li N, Li AP. Effects of culture duration on gene expression of $\mathrm{P} 450$ isoforms, uptake and efflux transporters in primary hepatocytes cultured in the absence and presence of interleukin-6: implications for experimental design for the evaluation of downregulatory effects of biotherapeutics. Curr Drug Metab. 2012;13(7):938-946. doi:10.2174/138920012802138570

18. Yu H, Kortylewski M, Pardoll D. Crosstalk between cancer and immune cells: role of STAT3 in the tumour microenvironment. Nat Rev Immunol. 2007;7(1):41-51. doi:10.1038/nri1995

19. Yu H, Lee H, Herrmann A, Buettner R, Jove R. Revisiting STAT3 signalling in cancer: new and unexpected biological functions. Nat Rev Cancer. 2014;14(11):736-746. doi:10.1038/nrc3818

20. Bene MC, Castoldi G, Knapp W, et al. Proposals for the immunological classification of acute leukemias. European group for the immunological characterization of leukemias (EGIL). Leukemia. 1995;9(10):1783-1786.

21. Gonzalez Garcia JR, Meza-Espinoza JP. Use of the international system for human cytogenetic nomenclature (ISCN). Blood. 2006;108(12):3952-3953. author reply 3953. doi:10.1182/blood2006-06-031351

22. Gao C, Zhao XX, Li WJ, et al. Clinical features, early treatment responses, and outcomes of pediatric acute lymphoblastic leukemia in China with or without specific fusion transcripts: a single institutional study of 1004 patients. Am J Hematol. 2012;87(11):1022-1027. doi:10.1002/ajh.23307

23. Chakraborty D, Sumova B, Mallano T, et al. Activation of STAT3 integrates common profibrotic pathways to promote fibroblast activation and tissue fibrosis. Nat Commun. 2017;8(1):1130. doi:10.1038/ s41467-017-01236-6

24. Norelli M, Camisa B, Barbiera G, et al. Monocyte-derived IL-1 and IL-6 are differentially required for cytokine-release syndrome and neurotoxicity due to CAR T cells. Nat Med. 2018;24(6):739-748. doi:10.1038/s41591-018-0036-4

25. Wu R, Liu X, Yin J, et al. IL-6 receptor blockade ameliorates diabetic nephropathy via inhibiting inflammasome in mice. Metabolism. 2018;83:18-24. doi:10.1016/j.metabol.2018.01.002

26. Guo P, Wang X, Liu L, Belinsky MG, Kruh GD, Gallo JM. Determination of methotrexate and its major metabolite 7-hydroxymethotrexate in mouse plasma and brain tissue by liquid chromatography-tandem mass spectrometry. J Pharm Biomed Anal. 2007;43(5):1789-1795. doi:10.1016/j.jpba.2006.12.034

27. Morgan ET, Goralski KB, Piquette-Miller M, et al. Regulation of drug-metabolizing enzymes and transporters in infection, inflammation, and cancer. Drug Metab Dispos. 2008;36(2):205-216. doi:10.1124/dmd.107.018747

28. Merrell MD, Nyagode BA, Clarke JD, Cherrington NJ, Morgan ET. Selective and cytokine-dependent regulation of hepatic transporters and bile acid homeostasis during infectious colitis in mice. Drug Metab Dispos. 2014;42(4):596-602. doi:10.1124/ dmd.113.055525

29. Yang J, Yan B. Photochemotherapeutic agent 8-methoxypsoralen induces cytochrome P450 3A4 and carboxylesterase HCE2: evidence on an involvement of the pregnane X receptor. Toxicol Sci. 2007;95(1):13-22. doi:10.1093/toxsci/kfl120 
30. Kawauchi S, Nakamura T, Horibe S, et al. Down-regulation of hepatic CYP3A1 expression in a rat model of indomethacin-induced small intestinal ulcers. Biopharm Drug Dispos. 2016;37(9):522-532. doi:10.1002/ bdd. 2042

31. Teng S, Piquette-Miller M. Regulation of transporters by nuclear hormone receptors: implications during inflammation. Mol Pharm. 2008;5(1):67-76. doi:10.1021/mp700102q
32. Denson LA, Bohan A, Held MA, Boyer JL. Organ-specific alterations in RAR alpha: rXRalpha abundance regulate rat Mrp2 (Abcc2) expression in obstructive cholestasis. Gastroenterology. 2002;123 (2):599-607. doi:10.1053/gast.2002.34758

\section{Publish your work in this journal}

The Journal of Inflammation Research is an international, peerreviewed open-access journal that welcomes laboratory and clinical findings on the molecular basis, cell biology and pharmacology of inflammation including original research, reviews, symposium reports, hypothesis formation and commentaries on: acute/chronic inflammation; mediators of inflammation; cellular processes; molecular mechanisms; pharmacology and novel anti-inflammatory drugs; clinical conditions involving inflammation. The manuscript management system is completely online and includes a very quick and fair peerreview system. Visit http://www.dovepress.com/testimonials.php to read real quotes from published authors. 